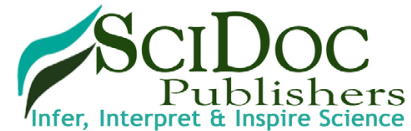

\title{
International Journal of Surgery and Research (IJSR)
}

ISSN 2379-156X

\section{Colorectal Anastomotic Leakage: A new, Validated Rat Model}

Research Article

Kevin WY van Barneveld ${ }^{1}$, Konstantinos A Vakalopoulos ${ }^{2}$, Joanna WAM Bosmans ${ }^{1}$, Ruben RM Vogels ${ }^{1}$, Geesien SA Boersema ${ }^{2}$, Marion JJ Gijbels ${ }^{3,4,5}$, Jack PM Cleutjens ${ }^{4}$, Johannes Jeekel ${ }^{2}$, Johan F Lange ${ }^{2}$, Nicole D Bouvy ${ }^{1 *}$

${ }^{1}$ Department of Surgery, Research Institute NUTRIM, Maastricht University Medical Centre, AZ, Maastricht, The Netherlands.

${ }^{2}$ Department of Surgery, Erasmus University Medical Centre Rotterdam, CA, Rotterdam, the Netherlands.

${ }^{3}$ Department of Molecular Genetics, Research Institute CARIM, Maastricht University Medical Centre, AZ, Maastricht, The Netherlands.

${ }^{4}$ Department of Pathology, Research Institute CARIM, Maastricht University Medical Centre, AZ, Maastricht, The Netherlands.

${ }^{5}$ Department of Medical Biochemistry, Academic Medical Centre, Meibergdreef AZ, Amsterdam, The Netherlands.

\section{Abstract}

Background: Anastomotic leakage (AL) remains an important complication after colorectal surgery. Experimental research aims to find a solution to overcome this serious complication; however, no validated AL model exists in the rat. This study was designed to develop a feasible new, reproducible AL model for use in colorectal anastomotic research.

Methods: Forty-four male Wistar rats, randomly divided into 4 groups, underwent a midline laparotomy and transection of the proximal colon. An anastomosis was created with 3, 4, 5, or 12 (control) sutures. After 7 days, the rats were euthanized and scored for the presence of AL. The secondary outcomes were anastomotic bursting pressure (ABP), histological evaluation, and maturation of collagen. After the initial experiment, the AL model was reproduced in an external academic center to ensure reproducibility.

Results: AL occurred in $68.8 \%$ of rats treated with an insufficient anastomosis, compared to $10 \%$ of the control group $(\mathrm{P}=0.005)$. AL rates were highest $(81.8 \%)$ in the 3 -suture group and lowest $(50 \%)$ when 5 sutures were used. The experiment with 4 sutures was reproduced at an external academic center and showed similar AL rates as in our center $(72.7 \%$ vs. $63.6 \%)$.

Conclusions: Our rat model for inducing AL by creating an anastomosis using an insufficient number of sutures is feasible and reproducible. When combining all outcome measures, the AL model for colorectal leakage using 4 sutures is the most suitable for use in future research.

Keywords: Anastomosis; Animal Model; Bowel; Colonic Anastomotic Leakage; Experimental; Rodent.

\section{Introduction}

In colorectal surgery, anastomotic leakage (AL) is known to be a frequent and life-threatening complication. The literature reports incidences of 5 to $17 \%$ of cases [1-3]. AL is associated with morbidity and mortality: AL patients undergo more radiological diagnostics, need prolonged Intensive Care Unit (ICU) admission, require more re-operations, require the construction of stomas, and, although debated, might even suffer from higher local and distant oncological recurrence rates and decreased long-term survival [4-9].

During the past decades, much research has been carried out to overcome the problem of AL. Part of this research is focused on the identification of risk factors, prediction, and diagnostics for anastomotic leakage $[3,10,11]$ whereas other studies focus on developing new techniques to improve anastomotic strength or healing [12-14]. This latter research often includes the use of

\footnotetext{
*Corresponding Author:

Nicole D Bouvy MD, PhD

Professor, Department of Surgery, Research Institute NUTRIM, Maastricht University Medical Centre, PO Box 5800, 6202 AZ, Maastricht, the Netherlands.

Tel: +31433875492

Fax: +31433875473

E-mail: n.bouvy@mumc.nl

Received: February 12, 2016

Accepted: December 22, 2016

Published: December 27, 2016

Citation: Kevin WY van Barneveld, Konstantinos A Vakalopoulos, Joanna WAM Bosmans, Ruben RM Vogels, et al., (2016) Colorectal Anastomotic Leakage: A new, Validated Rat Model. Int J Surg Res. 3(6), 61-67. doi: http://dx.doi.org/10.19070/2379-156X-1600013

Copyright: Nicole D Bouvy ${ }^{\circ}$ 2016. This is an open-access article distributed under the terms of the Creative Commons Attribution License, which permits unrestricted use, distribution and reproduction in any medium, provided the original author and source are credited.
} 
animal models for AL. Unfortunately, a wide variety of animal models are in use, mainly for the testing of tissue adhesives in the bowel [15-18], internal devices [19-21], or systemic therapies [22]. This large variety of AL models seriously impairs the possibility of adequately comparing outcomes between studies, making it difficult for new therapies to progress into the clinical testing phase [23]. Furthermore, most studies use primary outcomes like anastomotic bursting pressure (ABP), breaking strength or histological outcomes to define anastomotic healing; outcomes which cannot be measured in leaking anastomoses and do not necessarily predict the onset of AL [24-27].

Only a few studies have been designed specifically to develop a standardized animal model for use in research regarding AL [25, 28-30]. Komen and colleagues introduced a new experimental mouse model and provided information about its reproducibility [25]. However, most present-day AL research is performed in the rat, as the mouse has been found to be too small to adequately test anastomotic healing/sealing devices such as external applications to enhance wound healing, or tissue adhesives to reinforce anastomotic strength [18-31]. For the purpose of anastomotic testing, there is a need for a low-cost, easy to use, and reproducible animal model.

Therefore, in this study, we aim to evaluate the feasibility and reproducibility of a new experimental model of AL induced by standardized technically insufficient anastomoses in rats.

\section{Materials and Methods}

Male Wistar rats weighing 250-350g were housed and cared for at the Central Animal Facility of the Maastricht University Medical Centre, the Netherlands. Standard rat chow and water were supplied ad libitum, according to local standards. Similar to our local human pre-operative procedures, no bowel preparation or irrigation was performed. The experimental protocol complied with the Dutch Animal Experimentation Act and was approved by the local Animal Experimental Committee.

\section{Anesthesia and Surgical Procedures}

Anesthesia was induced by the inhalation of isoflurane $5.0 \mathrm{vol} \%$ (Forene, Abbott Laboratories, USA), followed by a maintenance dose of $2.5 \mathrm{vol} \%$. The animals received buprenorphine $0.1 \mathrm{mg} / \mathrm{kg}$ (Temgesic, Schering-Plough, USA) pre-operatively for analgesia. During the surgical procedure, the animals were placed on a temperature-controlled heating pad. The abdominal skin was shaved, disinfected with iodine $1 \%$ and covered with sterile drapes. Experienced researchers, certified for animal research, performed all surgical procedures.

The abdominal cavity was accessed through a $5 \mathrm{~cm}$ midline incision. The cecum was identified and placed on sterile gauzes hydrated with sterile saline solution to prevent dehydration. The colon was transected $2 \mathrm{~cm}$ distally to the cecum, taking care not to damage the mesenteric vessels. An end-to-end inverting anastomosis was created using evenly distributed polypropylene 6/0 sutures (Prolene, Ethicon, Johnson \& Johnson, USA), after which the intestines were repositioned into the abdominal cavity. For postoperative hydration, a bolus of $5 \mathrm{~mL}$ sterile saline solution $\left(37^{\circ} \mathrm{C}\right)$ was injected into the abdominal cavity prior to the closure of the abdominal muscle layers with a running suture of polyglactine 4/0 (Vicryl, Ethicon, Johnson \& Johnson, USA). The skin was closed intracutaneously with a running suture of polyglecaprone 4/0 (Monocryl, Ethicon, Johnson \& Johnson, USA). After the operation, moist rat chow was available to enhance postoperative feeding and to reduce animal discomfort. Daily check-ups of all animals were carried out to evaluate animal welfare, and in cases of severe distress/illness, additional analgesics (buprenorphine) were administered to diminish discomfort. In the experimental protocol, discomfort endpoints were pre-defined, partly adapted from Roughan \& Flecknell [32]. Once these endpoints were reached, the rats would have to be euthanized preliminarily.

\section{Study Design}

This study consisted of 3 intervention groups in which a technically insufficient end-to-end colonic anastomosis was created using 3, 4 or 5 sutures. The control group received 12 equidistant sutures, which is considered a sufficient anastomosis and technically feasible without compromising reproducibility. A leakage rate of $12 \%$ was expected in the control group (based on prior research) and based on an expected difference of $48 \%$ in an ideal group, an alpha of 0.05 and power of 0.8 , a total of 10 rats per group were required. Because previous research showed a dropout rate of $10 \%$, the group size was estimated at 11 rats per group. Therefore, atotal of 44 rats were divided randomly into these 4 groups (Table 1). The goal of this experiment was to identify the amount of sutures needed to create an anastomosis leading to a leakage rate of $70 \%$, considered the best suitable rate for use in models in comparison studies. This high percentage reduces the number of animals needed to reach significance, without compromising the level of animal discomfort, dropouts, or financial means.

The primary outcome of this study was the percentage of AL; the secondary outcome measures were anastomotic bursting pressure, histological scoring, and the maturation of collagen.

\section{Macroscopic Evaluation}

Because the majority of AL occurs in the first week following surgery and gastrointestinal healing measured by bursting pressure will be at its maximum after 7 days [33], this period was chosen for follow-up. After 7 days, the rats were euthanized by an overdose of inhaled carbon dioxide. Thereafter, a re-laparotomy was performed and signs of anastomotic leakage were scored intra-abdominally. The Hinchey classification was used to score anastomotic leakage, ranging from Hinchey I (the presence of localized abscess para-colonic), Hinchey II (the presence of pelvic abscess), Hinchey III (purulent peritonitis: the presence of pus in the abdominal cavity) to Hinchey IV (feculent peritonitis: the presence of pus and feces in the abdominal cavity). AL was defined as the manifestation of fecal or purulent peritonitis (Hinchey III-IV) or the presence of one or more abscesses in the abdominal cavity (Hinchey I-II) [34].

Next, signs of bowel obstruction were scored since this was a frequently observed side effect in previous anastomotic leakage research $[13,15]$. Furthermore, adhesion formation to the anastomotic region was scored using the Zühlke scoring system [35]. 
Subsequently, anastomotic bursting pressure (ABP) was measured: the colon distal to the anastomosis was clamped, a plastic tube was inserted into the proximal end, and ligated with a single polyglactine 4/0 suture. Thereafter, the abdomen was filled with sterile saline solution and the pressure was gradually increased using a pressure device (IDEE, Maastricht, the Netherlands). $\mathrm{ABP}$ was monitored using a digital manometer until there were clear signs of air leakage: i.e. the presence of air bubbles in the saline solution $[15,19]$. The anastomotic segment was resected and prepared for histological evaluation.

\section{Microscopic Evaluation}

Specimens were fixated in formaldehyde 4\%, after which they were dehydrated using increasing concentrations of ethanol prior to being embedded in paraffin. After paraffin embedding, tissue sections $4 \mu \mathrm{m}$ thick were prepared and these tissue sections were stained with hematoxylin and eosin, according to standard histological protocols. Semiquantitative scoring was used to evaluate the inflammatory response: not present, slightly present, moderately present or abundantly present [34].

To evaluate collagen formation, tissue sections were stained with Picrosirius red [36]. When Picrosirius red staining is combined with digital imaging, different types of collagen can be identified and quantified. A red color indicates thick and mature collagen type 1 fibers and green indicates thin immature collagen type 3 fibers. Sections were exposed to a $0.1 \%$ solution of Sirius red in saturated aqueous picric acid for 90 minutes followed by 2 minutes of washing in $0.01 \mathrm{~N} \mathrm{HCl}$, dehydration and mounting with entellan. Images of the (peri) anastomotic region were taken at a magnification of 200x using cross-polarization light microscopy (Leica DM5000B, Leica Microsystems, Switzerland). The percentages of collagen in the anastomotic tissue were calculated. The maturity level of the collagen was estimated by calculating the red versus green area ratio using the Qwin morphometry-system (Leica QWin V3.5.1, Leica Microsystems) $[15,19]$. Three randomly chosen images per tissue sample were analyzed per group. An experienced animal pathologist, who was blinded to the identity of the groups, microscopically evaluated all samples in a randomized matter.

\section{The External Test for Reproducibility}

In the surgical research facilities of the Erasmus Medical Center in Rotterdam, we performed an additional experiment with 11 rats, using the exact same operative procedure. During the operation, performed by a researcher not present during the earlier experiments (GB), the construction of an anastomosis was performed using 4 sutures, which was the best performing interventional group. After 7 days of follow up, the same experimental protocol as above was used to evaluate the primary outcomes.

\section{Statistical Analysis}

All data are expressed as the means with the standard error of the mean (SEM). Differences were analyzed using one-way ANOVA and t-tests. A Bonferroni corrected P-value $<0.05$ was considered statistically significant. All analyses were performed using IBM SPSS Statistics, version 21.0 for Mac (IBM SPSS, USA). All graphs were composed using GraphPad Prism, version 5.0 a for Mac (GraphPad Software, USA).

\section{Results}

At baseline, the rats weighed $308 \pm 3.0 \mathrm{~g}$, and no significant differences between groups were observed $(P=0.292$; Table 1). Perioperatively, a total of 2 animals died, 1 in the control group and 1 in the 5 -suture group, most likely due to respiratory depression following anesthesia. During follow up, no discomfort endpoints were reached. However, 1 animal in the 4-suture group died, and the postmortem examination showed bowel obstruction and AL. All other animals had normal postoperative recovery. After 7 days of follow up, the average weight was $285 \pm 3.1 \mathrm{~g}$, an average weight loss of $6.7 \pm 0.79 \%$. No significant differences between groups were observed.

\section{Clinical Outcomes}

After the 7-day follow-up period, significantly higher AL percentages were observed in the interventional groups compared to the control group: $68.8 \%$ vs. $10.0 \%, P=0.005$ (Figure 1 ). No significant differences were found between the 3 interventional groups: $81.8 \%, 72.7 \%$, and $50.0 \%$, for the 3 -, 4-, and 5 -suture groups, respectively.

In contrast to animals with AL, animals without the presence of AL showed no adhesions to the anastomotic region (Zühlke score 0$)$. Animals suffering from AL showed only mild to moderate adhesions to the anastomosis (Zühlke score 2-3), except for 1 animal with a total dehiscence of the anastomosis, in which massive adhesion formation (Zühlke score 4) and fecal peritonitis were observed (Table 2). Furthermore, comparable rates of postoperative bowel obstruction were observed in the interventional groups versus the control group: $36.3 \%$ vs. $10.0 \%$, $P=0.183$ (Table 2). Between the individual interventional groups,

Table 1. Baseline and Postoperative Weight Characteristics of Experimental Groups.

\begin{tabular}{|c|c|c|c|c|c|}
\hline & Control & 3 sutures & 4 sutures & 5 sutures & $\boldsymbol{P}$ - value \\
\hline Number of animals (n) & 11 & 11 & 11 & 11 & - \\
\hline Number of sutures & 12 & 3 & 4 & 5 & - \\
\hline Baseline weight (g) & $298 \pm 4.8$ & $313 \pm 5.9$ & $312 \pm 5.2$ & $308 \pm 7.6$ & 0.292 \\
\hline Endpoint weight (g) & $284 \pm 4.7$ & $292 \pm 6.7$ & $289 \pm 7.1$ & $276 \pm 5.7$ & 0.280 \\
\hline Weight loss (\%) & $4.0 \pm 0.7$ & $6.6 \pm 1.4$ & $6.9 \pm 1.5$ & $9.5 \pm 2.2$ & 0.117 \\
\hline
\end{tabular}

All measurements in this table are presented as mean $( \pm$ SEM) 
Figure 1. Anastomotic leakage (AL) rates per group after 7 days of follow up. Dark grey bars represent cases in which signs of AL were present at the time of euthanasia. After 7 days, significantly higher AL rates were found in the interventional groups compared to the control group. No significant differences were observed between the interventional groups. AL is defined by Hinchey I-IV scores.

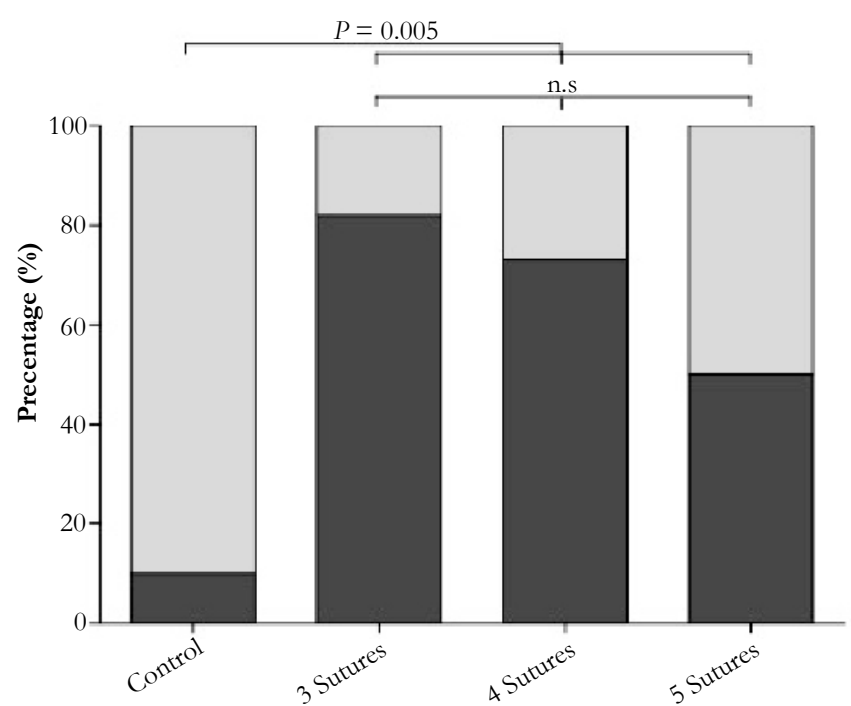

Table 2. Synopsis of Clinical Outcomes.

\begin{tabular}{|c|c|c|c|c|}
\hline & Control & 3 sutures & 4 sutures & 5 sutures \\
\hline Number of animals & $10^{*}$ & 11 & $10^{* * *}$ & $10^{*}$ \\
\hline Fecal peritonitis* & 0 & 1 (severe) & 0 & 1 (severe) \\
\hline Mechanical ileus* & 1 & 5 & 3 & 3 \\
\hline Adhesions total (mean) & 0.2 & 1.4 & 0.5 & 0.5 \\
\hline Median Zühlke score & 3 & 3 & 3 & 3 \\
\hline
\end{tabular}

* One rat in this group died following anesthesia-related complications

** One rat died during follow-up due to AL

$* * *$ Number of affected animals.

no significant differences were observed in the rates of bowel obstruction: $54.5 \%, 30 \%$, and $30 \%$ for the 3-, 4-, and 5-suture groups, respectively. All observed bowel obstructions were classified as mild, meaning a mild distention of the bowel segment.

\section{Anastomotic Bursting Pressure}

The bursting pressure analysis after 7 days showed a significant difference between the 3-suture group $(232.3 \pm 20.9 \mathrm{mmHg})$ and the control group (324.8 $\pm 24.4, P=0.024$, Figure 2$)$. Also, the 5 - suture group $(206.11 \pm 12.8)$ differed significantly from the control group $(P=0.004)$. The difference between the 4-suture group $(241.7 \pm 22.1)$ and the control group showed a trend towards significance $(P=0.074)$. No significant differences between the 3 interventional groups were observed.

\section{Histology}

The analysis of tissue sections stained for hematoxylin and eosin showed a well-organized anastomotic area in which clear differences were found between the extent of the inflammatory response in the control group compared to the 4-suture group (Figure 3). In the anastomotic region, no clear differences were observed between the different experimental groups $(3,4$, or 5 sutures) in terms of the amount of collagen, macrophages, granulocytes, and neoangiogenesis (data not shown). However, the control group had less inflammation and collagen deposition compared to the 4-suture experimental group. Picrosirius red staining of the anastomotic region showed comparable percentages of collagen in all groups $(P=0.339$; Table 3$)$. When focusing on collagen maturity, similar red/green ratios were observed for all groups, with the experimental groups having slightly more maturated collagen $(P=0.240)$.

\section{External Test for Reproducibility}

The 4-suture group was selected as the most feasible candidate for further reproducibility testing, with a leakage rate closest to the desired rate of $70 \%$. Furthermore, it showed comparable results in terms of secondary outcome measures. During the reproducibility testing at the Erasmus Medical Centre Rotterdam, an anastomotic leakage rate of $63.6 \%$ was observed. This rate is comparable with the leakage rate found in the 4-suture group during the earlier experiment (72.7\%). No animals died during the follow-up period. The postoperative recovery and weight loss were comparable to those observed in the initial experiment.

\section{Discussion}

In the present study we introduce a new, feasible, and reproducible 
Figure 2. Anastomotic bursting pressure (ABP) measured in situ after 7 days of follow up. The ABP measured in 2 of the interventional groups (3- and 5 sutures) was significantly lower compared to that of the control group. No significant differences were observed between the interventional groups.

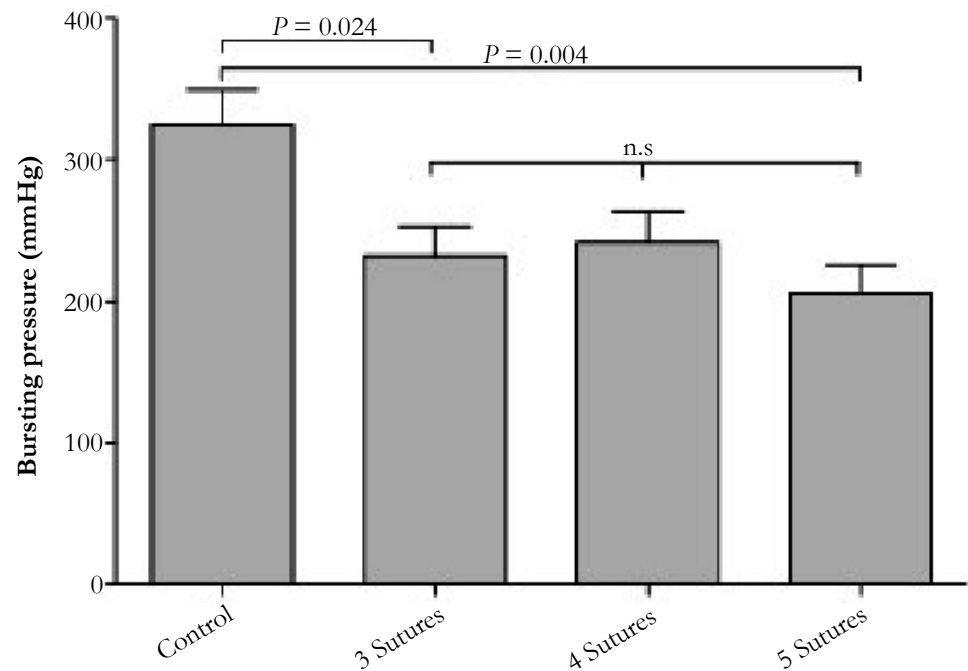

Figure 3. Semi-quantitative scoring of important aspects of wound healing, with more inflammation and granulocyte infiltration in the experimental groups (A), a representative image of the anastomotic site in the control group (B) and a representative anastomosis in the experimental group with 4 sutures $(\mathrm{C}) . * P=<0.05$.
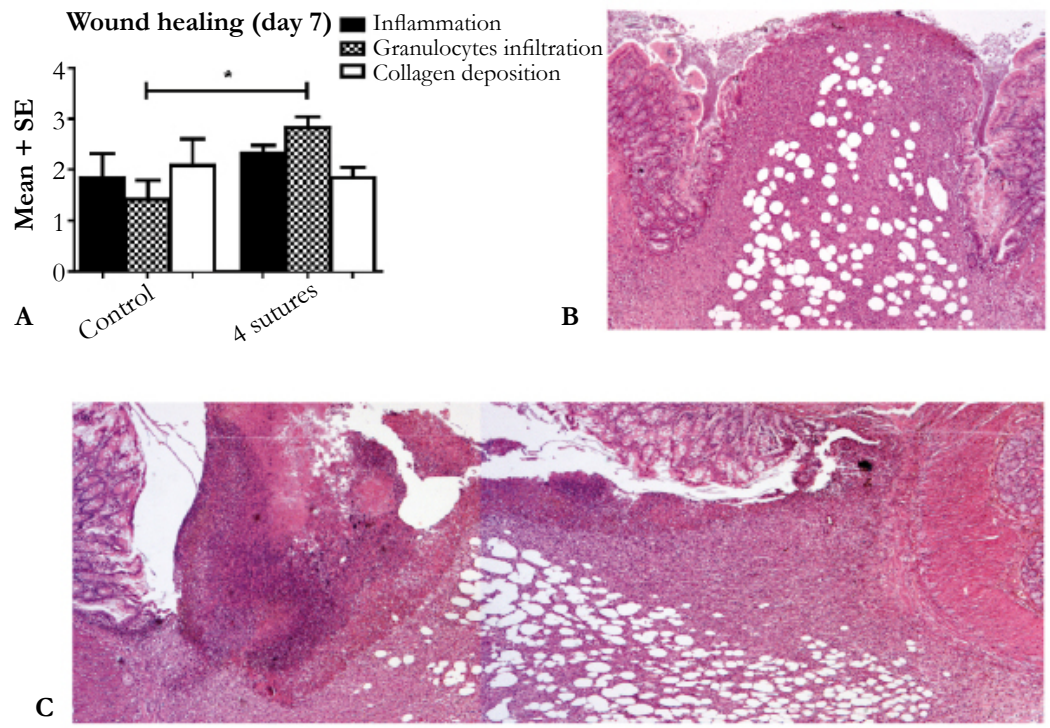

Table 3. Total Collagen and Mature/Immature Collagen Ratios.

\begin{tabular}{|c|c|c|c|c|c|}
\hline & Control & 3 sutures & 4 sutures & 5 sutures & P-value \\
\hline Collagen percentage $(\%)$ & $29.6 \pm 3.5$ & $22.4 \pm 2.1$ & $22.7 \pm 2.1$ & $25.4 \pm 4.1$ & 0.339 \\
\hline Red/green ratio & $5.56 \pm 0.72$ & $8.72 \pm 1.80$ & $6.40 \pm 1.30$ & $9.91 \pm 2.17$ & 0.240 \\
\hline
\end{tabular}

The relative collagen area was quantified as the percentage of total tissue surface. Maturity of collagen was estimated by calculating the $\mathrm{red} /$ green ratio. Red indicates thick, mature collagen. Green indicates thin, immature collagen. In terms of collagen percentage and $\mathrm{red} /$ green ratio, no significant differences were found between groups. All measurements in this table are presented as mean $( \pm$ SEM).

rat model for use in colorectal AL research. The model uses an insufficient number of sutures to create a leaking anastomosis. This model is easy to perform, achieves high percentages of AL, and was reproducible in an external academic center.

Although leaks in adults may occur due to a number of causes, many of which are patient-specific factors (i.e., gender, comorbidities, and medications), procedural factors (i.e., hypotension), or other technical issues (i.e., anastomotic ischemia) [37, 38], most AL models described in the literature are based on iatrogenic leakage, in which a partial defect or ischemia is created in the anastomosis and are considered models of colonic perforation [20, 21, 29, 30]. With these types of models, leakage rates of up to $100 \%$ have been described. Only a few models use a technically insufficient 
anastomosis with an insufficient number of sutures; however, such models have not been developed in rats [15, 18, 19, 25]. Clearly, in clinical situations a complete anastomosis is constructed; therefore, it was suggested that a model that develops dehiscence over time would be clinically more relevant [39]. Nonetheless, we believe that this model has its strengths in being artificial and standardized, making it more suitable for reproducing and for comparisons between different research centers.

In this study, we considered an AL rate of $70 \%$ most suitable for future colorectal AL research. With this percentage, fewer animals are needed to reach significant outcomes, which is ideal for studies regarding new leakage prevention strategies. Higher rates of AL, however, could be associated with a higher level of animal discomfort and possible death and are therefore not appropriate.

Several important characteristics are needed for a successful colorectal AL model. First of all, the anastomosis needs to be large enough to be suitable for anastomotic device testing, such as intraluminal stents or external sealing. Secondly, the colorectal tract should be comparable with that of humans, in terms of fecal consistency and gross anatomy. Furthermore, the costs should be low; larger animals are very costly, and a large numbers of animals are needed to achieve sufficient statistical power. The procedures should be easy to perform to minimize technical variability and to increase reproducibility. Finally, an optimal colorectal AL model should cause high percentages of AL to achieve a maximum effect per animal and to reduce the number of needed animals. To create an optimal rat colorectal AL model, we adapted parts of animal models used in earlier experiments from our research groups $[15,19,25]$. To overcome the problem observed by Van der Ham, who reported low levels of $\mathrm{AL}$ in an $\mathrm{AL}$ rat model with a left-sided anastomosis, we used a model with a right-sided anastomosis to obtain fecal consistency comparable to the human situation [16].

This experimental colorectal AL model clearly shows that the use of an insufficient number of sutures significantly increases the leakage rate from $10 \%$ (control) up to 50 to $82 \%$, depending on the number of sutures used. The lowest number of sutures (i.e., 3 sutures) showed the highest leakage rate, whereas the 4-and 5 -suture groups showed similar AL rates. The leakage rate from the control group is comparable with the clinical situation, where AL rates are reported to be as high as 5 to $17 \%[1,3]$. As discussed above, for an experimental model to be used in colorectal AL research, higher leakage rates around $70 \%$ are desirable, as a lower number of animals per group are needed to reach significance.

The group with the highest number of sutures (the 5-suture group) experience the highest amount of weight loss $(9.5 \%)$, whereas the 3 - and 4-suture groups did not show any differences $(6.6 \%$ and $6.9 \%$ ) compared to the control group. In terms of postoperative bowel obstruction, the 3-suture group had the highest rates (54\%), whereas the 4 - and 5 -suture groups did not show any differences. No differences between the interventional groups and control group were observed based on the histological examination. Furthermore, all interventional groups had significantly lower ABPs compared to the control group. Interestingly, the correlation analysis revealed no correlation between the ABP and AL rates, indicating that this measurement does not provide information on the onset of AL as observed by others [24-26].
Microscopically, clear differences were found between the control group (12 sutures) and the experimental groups. Leakage resulted in an evident larger inflammatory process that was evaluated histologically. No clear differences were found between the experimental groups, which is understandable because the semiquantitative scoring in animals that developed leakage is similar, probably due to the same amount of leakage. This might also explain why there were no differences in the collagen content and mature/immature collagen ratios between the experimental groups. However, due to the relatively high leakage rates, collagen content could have been used to discriminate between rats with and without AL, as recently described by Shogan and colleagues [40]. A big difference between their study and this work is that in this model no devascularization of mesenterial arteries was performed. Because it is known that adequate oxygen is critical for optimal collagen-crosslinking and synthesis [41], this could explain why no clear differences were found. In addition, the method of determining collagen ratios with Picrosirius red staining is perhaps not sufficiently sensitive enough to test subtle pathological changes that may have occurred in this model [42].

Repeating the experiment in an external academic center tested the reproducibility of this new colorectal AL model. This demonstrated the good reproducibility of the model, with comparable AL rates and postoperative recovery, indicating that the model may be valuable for use in various experimental centers. This study does have potential drawbacks. There is ongoing discussion about the comparability of physiological processes in rats and humans. For example, several exogenous factors differ between the human and experimental situation, which can limit the extrapolation of results to the human context. Because nonabsorbable polypropylene sutures have been shown to cause less tissue reaction as compared to absorbable sutures, we used non-absorbable sutures in our model, whereas in the majority of human cases, absorbable PDS sutures would have been used [43]. Although physiological processes in rats may not be a perfect reflection of the human situation, this validated model can be used to obtain results that provide a good indication of future outcomes in human subjects, especially regarding newly developed preventive measures such as sealants for AL.

In the present study, we introduce a new animal model for research regarding colorectal anastomotic leakage. This model, consisting of an end-to-end colonic anastomosis with 4 sutures, ensures a high onset of anastomotic leakage and has been proven to be reproducible. Therefore, this AL model may be useful in future experiments, in particular for testing novel colonic sealing devices to prevent AL.

\section{Acknowledgements and Declarations}

The authors would like to thank Mrs. L. Platenkamp, BSc for her contribution to data processing. The authors declare no conflicts of interest. Financial disclosure: None reported.

\section{References}

[1]. Krarup P-M, Nordholm-Carstensen A, Jorgensen LN, Harling H (2014) Anastomotic Leak Increases Distant Recurrence and Long-Term Mortality After Curative Resection for Colonic Cancer.: a nationwide cohort study. Ann Surgery. 259(5): 930-8.

[2]. Reinke CE, Showalter S, Mahmoud NN, Kelz RR (2013) Comparison of 
anastomotic leak rate after colorectal surgery using different databases. Dis Colon Rectum. 56(5): 638-44.

[3]. Trencheva K, Morrissey KP, Wells M, Mancuso CA, Lee SW, et al., (2013) Identifying important predictors for anastomotic leak after colon and rectal resection: prospective study on 616 patients. Ann Surg. 257(1): 108-13.

[4]. Kube R, Mroczkowski P, Granowski D, Benedix F, Sahm M, et al., (2010) Anastomotic leakage after colon cancer surgery: A predictor of significant morbidity and hospital mortality, and diminished tumour-free survival. Eur J Surg Oncol. 36(2): 120-4.

[5]. Ptok H, Marusch F, Meyer F, Schubert D, Gastinger I, Lippert H, et al., (2007) Impact of anastomotic leakage on oncological outcome after rectal cancer resection. Br J Surg. 94(12): 1548-54.

[6]. Hammond J, Lim S, Wan Y, Gao X, Patkar A (2014) The Burden of Gastrointestinal Anastomotic Leaks: an Evaluation of Clinical and Economic Outcomes. J Gastrointest Surg. 18(6): 1176-85.

[7]. Nachiappan S, Askari A, Malietzis G, Giacometti M, White I, et al., (2014) The Impact of Anastomotic Leak and Its Treatment on Cancer Recurrence and Survival Following Elective Colorectal Cancer Resection. World J Surg. 39(4): 1052-8.

[8]. Espín E, Ciga MA, Pera M, Ortiz H, Spanish Rectal Cancer Project (2015) Oncological outcome following anastomotic leak in rectal surgery. $\mathrm{Br} \mathrm{J}$ Surg. 102(4): 416-22.

[9]. Walker KG, Bell SW, Rickard MJFX, Mehanna D, Dent OF, et al., (2004) Anastomotic Leakage Is Predictive of Diminished Survival After Potentially Curative Resection for Colorectal Cancer. Ann Surg. 240(2): 255-9.

[10]. Reisinger KW, Poeze M, Hulsewé KWE, van Acker BA, van Bijnen AA, et al., (2014) Accurate Prediction of Anastomotic Leakage after Colorectal Surgery Using Plasma Markers for Intestinal Damage and Inflammation. J Am Coll Surg. 219(4): 744-51.

[11]. Komen N, Slieker J, Willemsen P, Mannaerts G, Pattyn P, et al., (2014) Acute phase proteins in drain fluid: a new screening tool for colorectal anastomotic leakage? The APPEAL study: analysis of parameters predictive for evident anastomotic leakage. Am J Surg. 208(3): 317-23.

[12]. Hoeppner J, Wassmuth B, Marjanovic G, Timme S, Hopt UT, et al., (2010) Anastomotic sealing by extracellular matrices (ECM) improves healing of colonic anastomoses in the critical early phase. J Gastrointest Surg. 14(6): 977-86.

[13]. Nordentoft T, Pommergaard HC, Rosenberg J, Achiam MP (2015) Fibrin Glue Does Not Improve Healing of Gastrointestinal Anastomoses: A Systematic Review. Eur Surg Res. 54(1-2): 1-13.

[14]. Vakalopoulos KA, Daams F, Wu Z, Timmermans L, Jeekel JJ, et al., (2013) Tissue adhesives in gastrointestinal anastomosis: a systematic review. J Surg Res. 180(2): 290-300.

[15]. Schreinemacher MH, Bloemen JG, Heijden SJ, Gijbels MJ, Dejong CH, et al., (2011) Collagen fleeces do not improve colonic anastomotic strength but increase bowel obstructions in an experimental rat model. Int J Colorectal Dis. 26(6): 729-35.

[16]. van der Ham AC, Kort WJ, Weijma IM, Jeekel H (1993) Transient protection of incomplete colonic anastomoses with fibrin sealant: an experimental study in the rat. J Surg Res. 55(3): 256-60.

[17]. Pantelis D, Beissel A, Kahl P, Wehner S, Vilz TO, et al., (2010) The effect of sealing with a fixed combination of collagen matrix-bound coagulation factors on the healing of colonic anastomoses in experimental high-risk mice models. Langenbecks Arch Surg. 395(8): 1039-48.

[18]. Slieker JC, Vakalopoulos KA, Komen NA, Jeekel J, Lange JF (2013) Prevention of leakage by sealing colon anastomosis: experimental study in a mouse model. J Surg Res. 184(2): 819-24.

[19]. Bloemen JG, Schreinemacher MH, de Bruine AP, Buurman WA, Bouvy ND, et al., (2010) Butyrate enemas improve intestinal anastomotic strength in a rat model. Dis Colon Rectum. 53(7): 1069-75.

[20]. Wang Y, Cai X, Jin R, Liang Y, Huang D, et al., (2011) Experimental Study of Primary Repair of Colonic Leakage with a Degradable Stent in a Porcine Model. J Gastrointest Surg. 15(11): 1995-2000.

[21]. Tsereteli Z, Sporn E, Geiger TM, Cleveland D, Frazier S, et al., (2008) Placement of a covered polyester stent prevents complications from a colorectal anastomotic leak and supports healing: randomized controlled trial in a large animal model. Surgery. 144(5): 786-92.
[22]. Tingstedt B, Nehéz L, Lindman B, Andersson R (2007) Effect of Bioactive Polypeptides on Leaking Large Bowel Anastomosis and Intestines in the Rat. J Invest Surg. 20(4): 229-35.

[23]. Yauw STK, Wever KE, Hoesseini A, Ritskes-Hoitinga M, van Goor H (2015) Systematic review of experimental studies on intestinal anastomosis. Br J Surg. 102(7): 726-734.

[24]. Månsson P, Zhang XW, Jeppsson B, Thorlacius H (2002) Anastomotic healing in the rat colon: comparison between a radiological method, breaking strength and bursting pressure. Int J Colorectal Dis. 17(6): 420-5.

[25]. D NKM, van der Wal H-C, Ditzel M, D G-JKP, Jeekel H, et al., (2009) Colorectal Anastomotic Leakage: A New Experimental Model. J Surg Res. 155(1): 7-12.

[26]. van der Vijver RJ, van Laarhoven CJHM, Ben M de Man, Lomme RMLM, Hendriks T (2012) The effect of fibrin glue on the early healing phase of intestinal anastomoses in the rat. Int J Colorectal Dis. 27(8): 1101-7.

[27]. Rushfeldt CF, Sveinbjørnsson B, Søreide K, Vonen B (2011) Risk of anastomotic leakage with use of NSAIDs after gastrointestinal surgery. Int J Colorectal Dis. 26(12): 1501-9.

[28]. Wenger FA, Szucsik E, Hoinoiu BF, Ionac M, Walz MK, et al., (2013) A new anastomotic leakage model in circular double stapled colorectal anastomosis after low anterior rectum resection in pigs. J Invest Surg. 26(6): 364-72.

[29]. Hoeppner J, Crnogorac V, Hopt UT, Weiser H-F (2009) The Pig as an Experimental Model for Colonic Healing Study of Leakage and Ischemia in Colonic Anastomosis. J Invest Surg. 22(4): 281-5.

[30]. Nordentoft T, Sørensen M (2007) Leakage of colon anastomoses: development of an experimental model in pigs. Eur Surg Res. 39(1): 14-6.

[31]. Wu Z, Vakalopoulos KA, Boersema GSA, Kroese LF, Lam KH, et al., (2014) The prevention of colorectal anastomotic leakage with tissue adhesives in a contaminated environment is associated with the presence of anti-inflammatory macrophages. Int J Colorectal Dis. 29(12): 1507-16.

[32]. Roughan JV, Flecknell PA (2001) Behavioural effects of laparotomy and analgesic effects of ketoprofen and carprofen in rats. Pain. 90(1-2): 65-74.

[33]. Thompson SK, Chang EY, Jobe BA (2006) Clinical review: Healing in gastrointestinal anastomoses, part I. Microsurgery. 26(3): 131-6.

[34]. Hinchey EJ, Schaal PG, Richards GK (1978) Treatment of perforated diverticular disease of the colon. Adv Surg. 12: 85-109.

[35]. Zühlke HV, Lorenz EM, Straub EM, Savvas V (1990) Pathophysiology and classification of adhesions. Langenbecks Arch Chir Suppl II Verh Dtsch Ges Chir. 1009-16.

[36]. Rich L, Whittaker P (2005) Collagen and picrosirius red staining: a polarized light assessment of fibrillar hue and spatial distribution. Braz J Morphol Sci. 22(2): 97-104.

[37]. Midura EF, Hanseman D, Davis BR, Atkinson SJ, Abbott DE, et al., (2015) Risk Factors and Consequences of Anastomotic Leak After Colectomy: a national analysis. Dis Colon Rectum. 58(3): 333-8.

[38]. Bakker IS, Grossmann I, Henneman D, Havenga K, Wiggers T (2014) Risk factors for anastomotic leakage and leak-related mortality after colonic cancer surgery in a nationwide audit. Br J Surg. 101(4): 424-32.

[39]. van der Vijver RJ, van Laarhoven CJHM, de Man BM, Lomme RMLM, Hendriks T (2013) Perioperative Pain Relief by a COX-2 Inhibitor Affects Ileal Repair and Provides a Model for Anastomotic Leakage in the Intestine. Surg Innov. 20(2): 113-8.

[40]. Shogan BD, Belogortseva N, Luong PM, Zaborin A, Lax S, et al., (2015) Collagen degradation and MMP9 activation by Enterococcus faecalis contribute to intestinal anastomotic leak. Sci Transl Med. 7(286): 286ra68-8.

[41]. Kologlu M, Yorganci K, Renda N, Sayek I (2000) Effect of local and remote ischemia-reperfusion injury on healing of colonic anastomoses. Surgery. 128(1): 99-104

[42]. Osman OS, Selway JL, Harikumar PE, Stocker CJ, Wargent ET, et al., (2013) A novel method to assess collagen architecture in skin. BMC Bioinformatics. 14(1): 260.

[43]. Schreinemacher MHF, van Barneveld KWY, Peeters E, Miserez M, Gijbels MJJ, et al., (2014) Adhesions to sutures, tackers, and glue for intraperitoneal mesh fixation: an experimental study. Hernia. 18(6): 865-72. 